\title{
Relationship between direct cortical stimulation and induced high-frequency activity for language mapping during SEEG recording
}

\author{
Pauline Cuisenier, MD, Bénédicte Testud, ${ }^{2}$ Lorella Minotti, MD, ${ }^{1,3}$ Samuel El Bouzaïdi Tiali, ${ }^{2}$ \\ Laurence Martineau, MD, ${ }^{1}$ Anne-Sophie Job, MD, ${ }^{1,3}$ Agnès Trébuchon, MD, PhD, ${ }^{4}$ \\ Pierre Deman, PhD, ${ }^{3}$ Manik Bhattacharjee, $\mathrm{PhD},{ }^{3}$ Dominique Hoffmann, MD, ${ }^{1}$ \\ Jean-Philippe Lachaux, PhD, 5,6 Monica Baciu, MD, PhD,, Philippe Kahane, MD, PhD, 1,4 and \\ Marcela Perrone-Bertolotti, $\mathrm{PhD}^{2,7}$
}

\begin{abstract}
1'Department of Neurology, CHU Grenoble Alpes, Grenoble; '2Université Grenoble Alpes, CNRS, LPNC UMR 5105, Grenoble; 3Université Grenoble Alpes, Institut des Neurosciences, GIN, Grenoble; ${ }^{4}$ Aix Marseille Université, INSERM, INS, Institut de

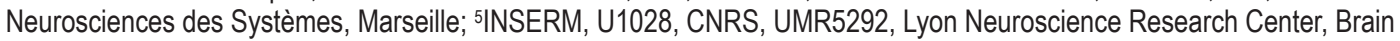
Dynamics and Cognition Team, DYCOG, Lyon; 'Université Lyon 1, Lyon, France; and ${ }^{7}$ Institut Universitaire de France
\end{abstract}

\begin{abstract}
OBJECTIVE The authors assessed the clinical relevance of preoperative task-induced high-frequency activity (HFA) for language mapping in patients with refractory epilepsy during stereoelectroencephalography recording. Although HFA evaluation was described as a putative biomarker of cognition, its clinical relevance for mapping language networks was assessed predominantly by studies using electrocorticography (ECOG).
\end{abstract}

METHODS Forty-two patients with epilepsy who underwent intracranial electrode implantation during both task-induced HFA and direct cortical stimulation (DCS) language mapping were evaluated. The spatial and functional relevance of each method in terms of specificity and sensitivity were evaluated.

RESULTS The results showed that the two methods were able to map classic language regions, and a large and bilateral language network was obtained with induced HFA. At a regional level, differences were observed between methods for parietal and temporal lobes: HFA recruited a larger number of cortical parietal sites, while DCS involved more cortical temporal sites. Importantly, the results showed that HFA predicts language interference induced by DCS with high specificity (92.4\%; negative predictive value $95.9 \%$ ) and very low sensitivity (8.9\%; positive predictive value $4.8 \%$ ).

CONCLUSIONS DCS language mapping appears to be more appropriate for an extensive temporal mapping than induced HFA mapping. Furthermore, induced HFA should be used as a complement to DCS to preselect the number of stimulated sites during DCS, by omitting those reported as HFA-. This may be a considerable advantage because it allows a reduction in the duration of the stimulation procedure. Several parameters to be used for each method are discussed and the results are interpreted in relation to previous results reported in ECOG studies.

https://thejns.org/doi/abs/10.3171/2020.2.JNS192751

KEYWORDS induced high-frequency activity; stereoelectroencephalography; direct cortical stimulation; language mapping; surgical technique

$\mathrm{E}$ PILEPSY surgery is one of the current treatments used to suppress drug-resistant seizures in epileptic patients. In this framework, preoperative stereoelectroencephalography (SEEG) exploration is performed in $25 \%-50 \%$ of patients and allows the identification of the epileptogenic zone to be removed and of the eloquent regions to be spared.$^{1-3}$ Although direct cortical stimulation
(DCS), classically performed during preoperative SEEG, is considered the "gold standard" method for functional mapping before surgery, it has several limitations: ${ }^{4,5} 1$ ) it is time-consuming and may lead to seizures, reducing the reproducibility of symptoms within and between patients; 2 ) it does not always induce robust clinical signs (especially if stimulation is applied on associative language cor-

ABBREVIATIONS BA = Brodmann's area; DCS = direct cortical stimulation; ECOG = electrocorticography; HFA = high-frequency activity; LEC = language experimental condition; MNI = Montreal Neurological Institute; NPV = negative predictive value; PPV = positive predictive value; SEEG = stereoelectroencephalography.

SUBMITTED October 10, 2019. ACCEPTED February 13, 2020.

INCLUDE WHEN CITING Published online April 24, 2020; DOI: 10.3171/2020.2.JNS192751. 
tices); 3) it may induce subtle behavioral symptoms difficult to detect or interpret (such as lexicosemantic deficits for language); and 4) a patient's subjective experience can be misleading, which adds difficulties for DCS interpretation. All of these reasons could explain why DCS may fail to reveal the functionality of a given brain region, revealing only a small part of the issue within a larger functional network. Moreover, despite SEEG information provided in presurgical evaluation, postoperative language deficits (such as anomia) may appear in some cases (see Hamberger ${ }^{6}$ ). Overall, the SEEG criteria used for identifying eloquent brain regions are not completely clear, and DCS during SEEG should be improved by combining it with other mapping methods.

An alternative method for mapping functional networks before surgery is the use of high-frequency activities (HFAs) ${ }^{7}$ induced by cognitive tasks. Broadband HFAs $(50-150 \mathrm{~Hz})$ have been proposed as putative biomarkers of cognitive processes ${ }^{7}$ and are currently considered as a proxy of population-level spiking activity while patients perform a cognitive task. Thus, many studies have reported that induced HFA is a promising functional mapping method $^{8}$ that could be at least complementary to DCS and overcome its limitations. For language in particular (which is especially affected in these patients ${ }^{9,10}$ ), induced HFA assessment can be a reliable method. ${ }^{8}$ Induced HFA provides high specificity for linguistic processes (such as word recognition, sentence comprehension, reading, and naming ${ }^{11-13}$ ) and subtle functional regional specificities (see Lachaux et al. ${ }^{8}$ for a review).

Several studies have compared induced HFA to DCS mapping, ${ }^{14}$ but few have explored this relationship with the SEEG method. ${ }^{15}$ In the present study, we evaluated the clinical relevance of the preoperative induced HFA language functional mapping and compared it to DCS mapping results during SEEG.

\section{Methods}

\section{Patient Selection and Population Studied}

Between 2010 and 2015, 95 epilepsy surgery candidates underwent SEEG recordings at Grenoble Alpes University Hospital (France), of whom 42 were selected based on the following inclusion criteria: 1) functional mapping with both DCS and HFA study during the SEEG investigation, 2) French-speaking, 3) older than 12 years of age, and 4) sufficient cognitive abilities to perform language tasks (as assessed by neuropsychological scores). Patients provided written informed consent and the study was approved by the National French Ethical Committee. The 42 patients underwent high-resolution MRI, scalp video-EEG monitoring, and neuropsychological evaluation before the SEEG procedure. Demographic and clinical characteristics of the studied population are given in Table 1.

\section{SEEG Study}

\section{SEEG Electrode Implantation}

Eleven to 18 semirigid multilead electrodes were implanted in each patient according to the robot-assisted SEEG electrode implantation technique currently in use at our institution. SEEG electrodes had a diameter of 0.8 $\mathrm{mm}$ and, depending on the target structure, consisted of $10-15$ contact leads $2 \mathrm{~mm}$ wide and $1.5 \mathrm{~mm}$ apart (i.e., 3.5 $\mathrm{mm}$ from center to center; DIXI Medical). The strategy of implantation was entirely based on clinical purposes and depended on the hypothesized origin of seizures, ${ }^{16}$ with no reference to the present study. Precise anatomical electrode location and display (and their Montreal Neurological Institute [MNI] coordinates) were obtained by the coregistration of preimplantation and postimplantation volumetric brain MR images using SPM12 (http://www.fil.ion.ucl. ac.uk/spm) and IntrAnat ${ }^{17}$ (IntrAnat Electrodes; available at https://f-tract.eu/index.php/software/) software.

\section{SEEG Recordings}

SEEG recordings were conducted using a video-SEEG monitoring system (Micromed), which allowed simultaneous data recording from 128 depth-EEG electrode sites. The data were bandpass filtered online from 0.1 to $200 \mathrm{~Hz}$ at a sampling frequency of $512 \mathrm{~Hz}$. Data were recorded using a reference electrode located in white matter, and the signal in each recording site was subsequently re-referenced with respect to its closest site (bipolar derivations). This bipolar montage (bipolar derivation) has a number of advantages over common referencing; notably, it reduces signal artifacts common to adjacent electrode contacts, such as the $50-\mathrm{Hz}$ main artifact or distant physiological artifacts, and it achieves a high local specificity by canceling out effects of distant sources that spread equally to both adjacent sites through volume conduction. The spatial resolution achieved by the bipolar SEEG was on the order of $3 \mathrm{~mm} .{ }^{2,18}$ Interictal and ictal analysis of SEEG traces was conducted visually.

\section{Direct Cortical Stimulation}

DCS was performed according to our routine procedure, ${ }^{1,19}$ during sessions that lasted 1-3 hours. The aim of DCS was to reproduce part of, or the totality of, the ictal semiology, and to localize eloquent functional areas that had to be spared for further surgery. Stimulations at $50 \mathrm{~Hz}$ were applied between two contiguous contacts at different levels along the axis of each electrode, chosen to be in the gray matter according to visual inspection of spontaneous SEEG signals. A bipolar montage was used to help eliminate the signal common to adjacent electrode contacts and to avoid artifacts coming from distant sources. Bipolar stimulation was delivered using a repetitive square-wave electric current of 1- to 3-msec pulse width designed for a safe diagnostic stimulation of the human brain (Micromed), according to parameters proven to produce no structural damage. The safety data from DIXI (used here) suggest a safe upper limit of charge density delivered to stimulated tissue of $50 \mu \mathrm{C} / \mathrm{cm}^{2}$. Charge density depends on stimulation intensity and pulse duration. The intensity used in our study involved stepwise increases ranging from 0.2 to $3 \mathrm{~mA}$ (until clinical subjective or objective responses, or afterdischarges or seizures, were obtained) and for a maximum duration of 5 seconds or less depending on the type of induced clinical response.

During DCS sessions, patients were asked to perform overt speech tasks, including counting and naming. Naming was performed using black-and-white drawings of 


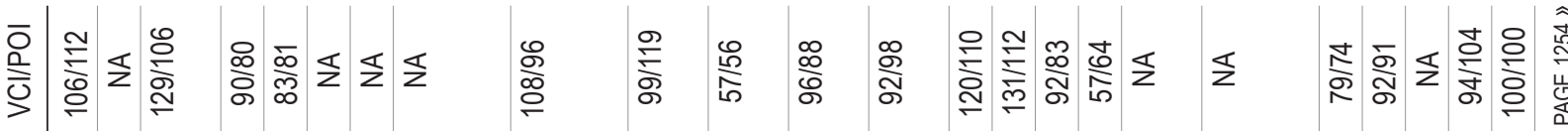

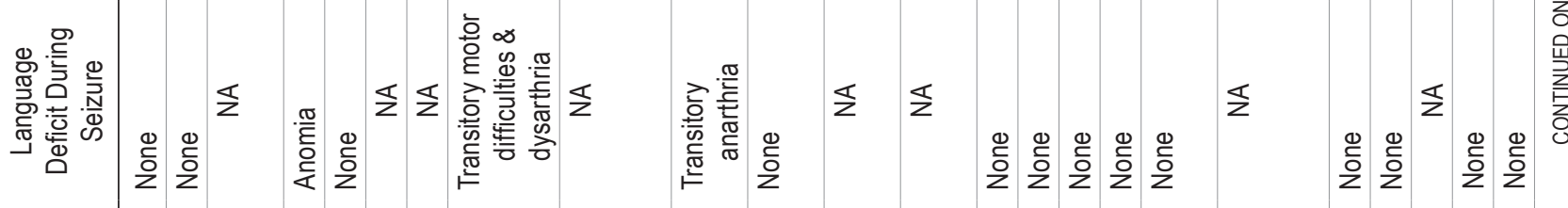

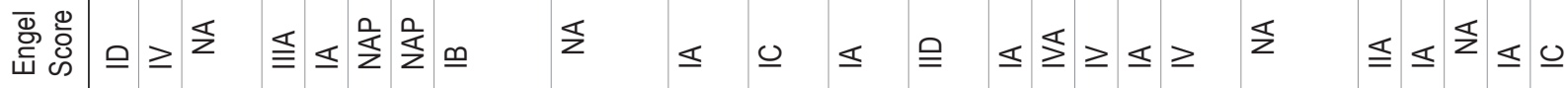

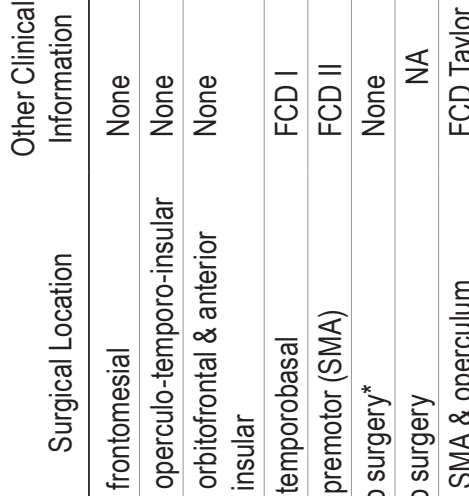

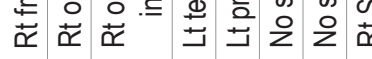

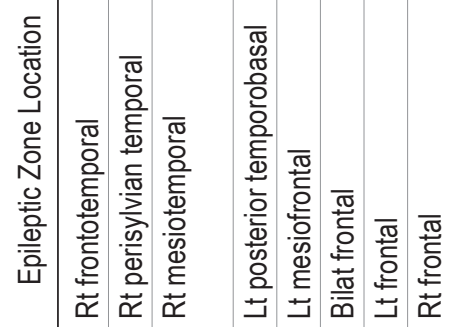

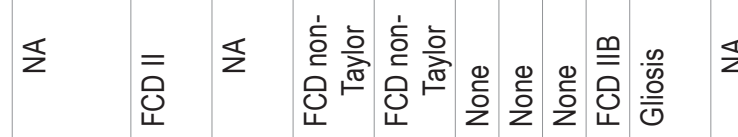

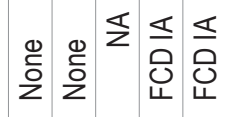
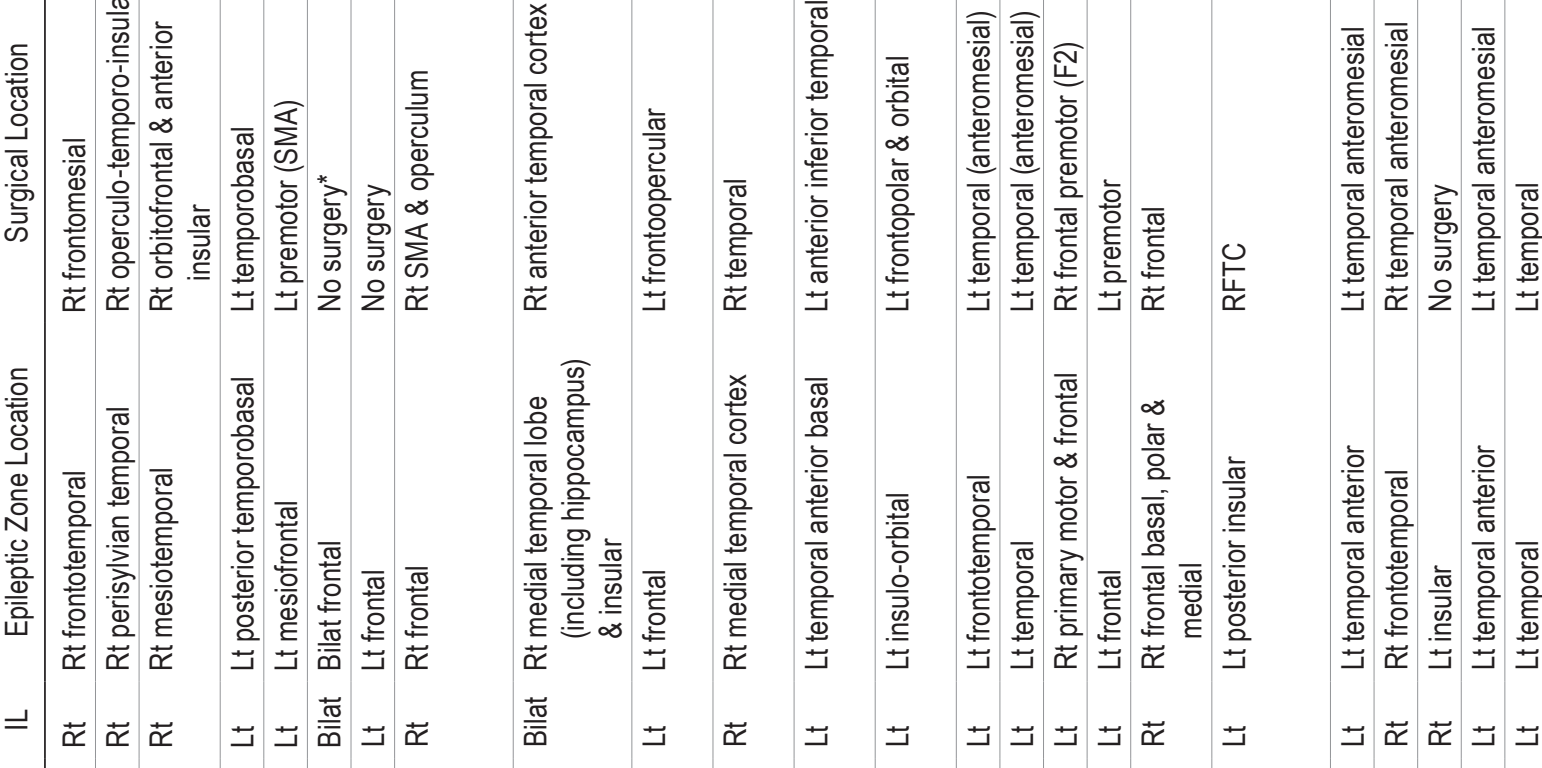

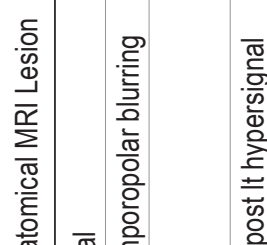

唁

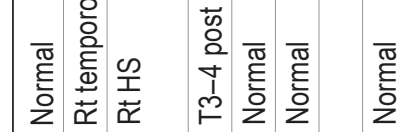
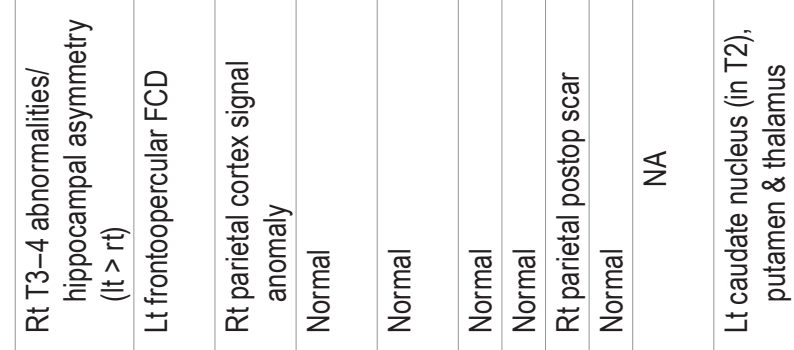

至

$\stackrel{5}{2}$

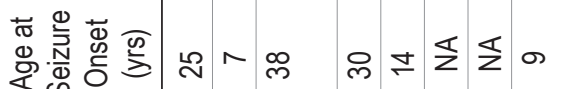

\&

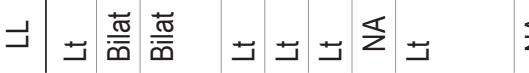

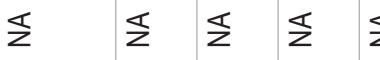
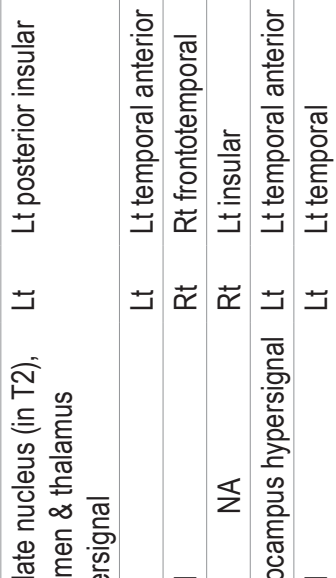

$\Xi \vec{\propto} \ddot{\propto} \Xi \sqsupset$

\section{总}

要 $\vec{x}$ वै $\vec{x} \vec{x} \vec{x} \vec{x} \vec{x}$

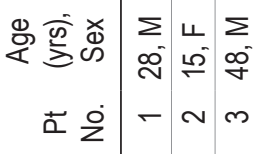

แ ᄂ $\Sigma \leftarrow \div$

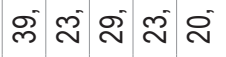

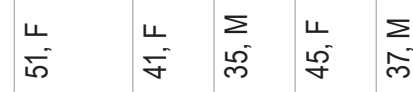

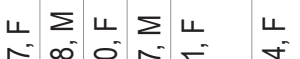

น ᄂ $\Sigma \leftarrow$

の 의 $\cong$

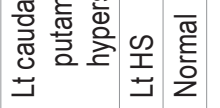

产

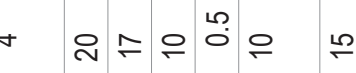

$\stackrel{n}{\sim} \sim=0$

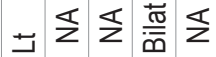

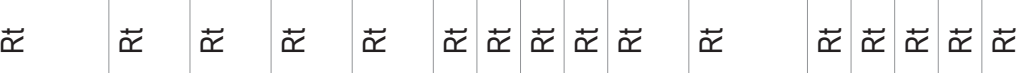




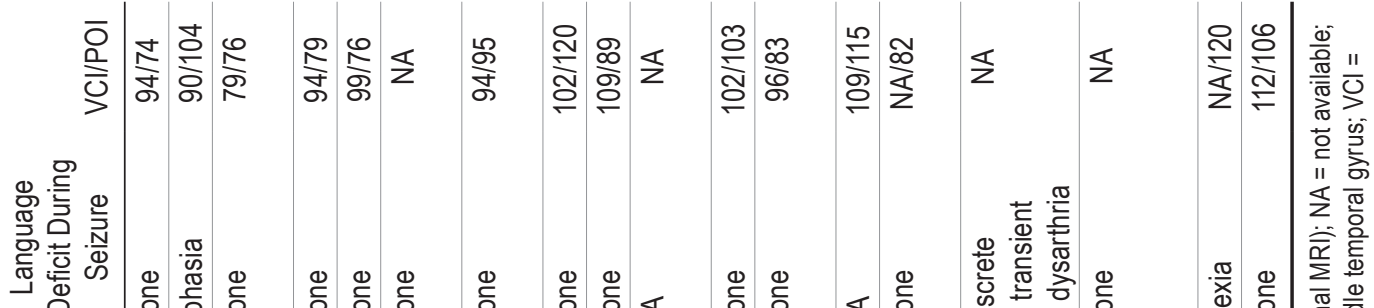

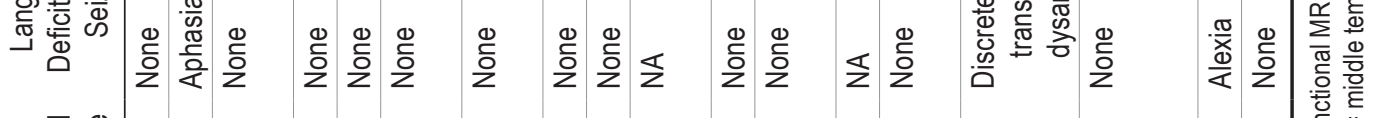

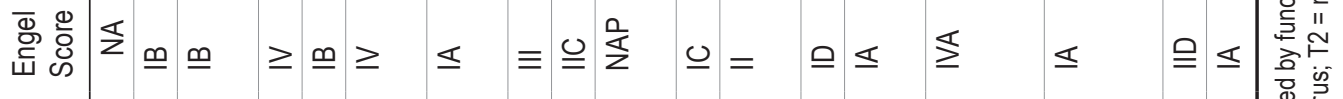

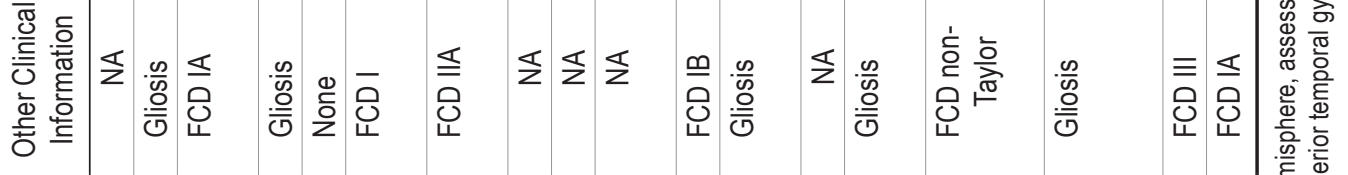

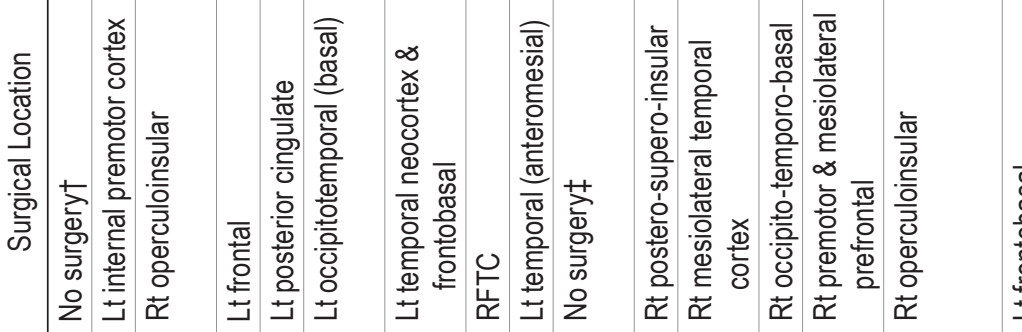

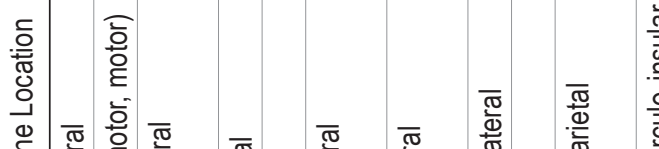

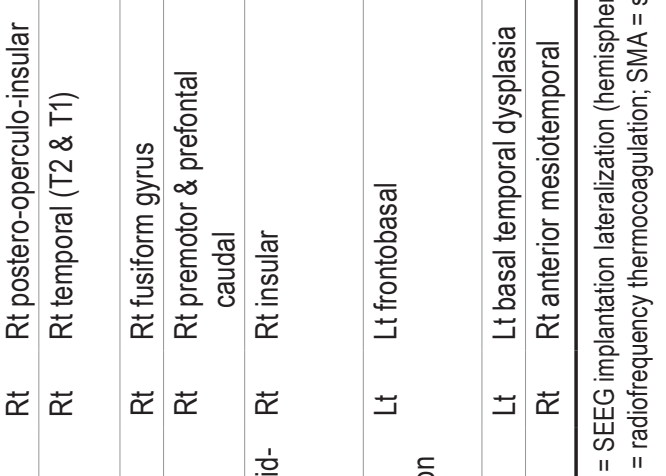

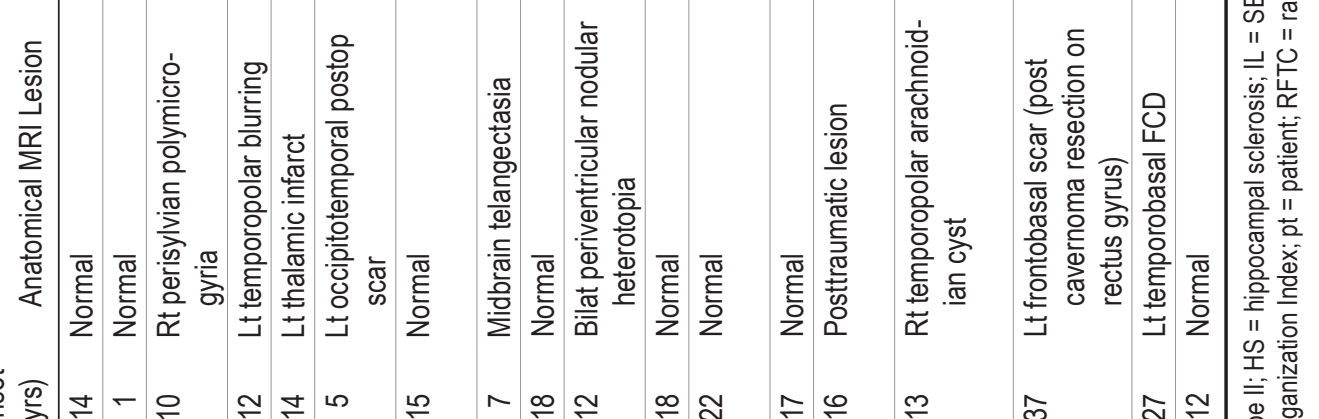

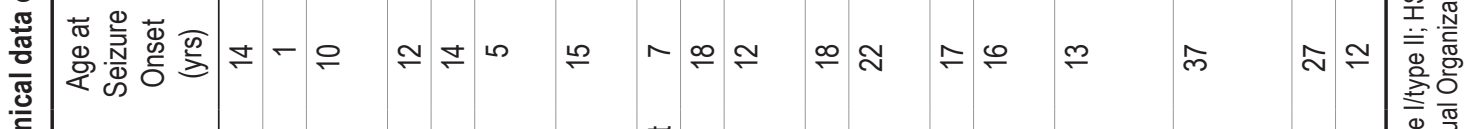

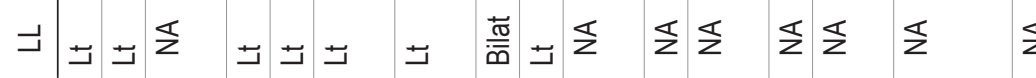

空

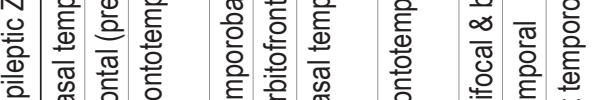

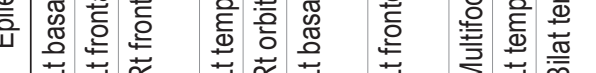

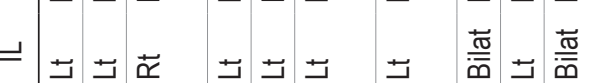

$\mathscr{\infty}$

离

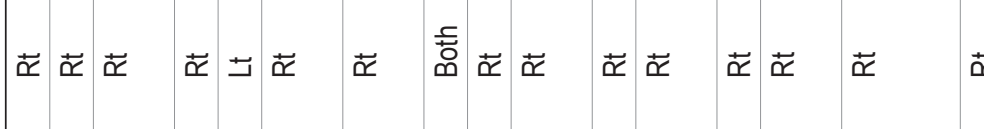

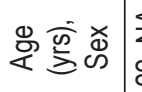

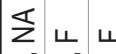

ㅁํำڤ

ч ᄂ ᄂ $\Sigma$

ᄂ ᄂ $\longleftarrow \leftleftarrows$

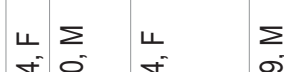

징

ฐิ

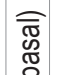

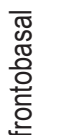

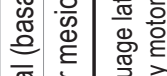

흔 은 홇 츈

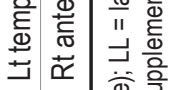


common objects from the French DO 80 task..$^{20}$ DCS was performed immediately after every speech task onset. DCS findings were classified by the examiner (neurologist; L. Minotti, A.S.J., or P.K.) and documented directly in patients' SEEG records.

\section{Language Task for Induced HFA Mapping}

Induced HFA language mapping was performed during SEEG recordings using a computerized language protocol (abbreviated "LEC" [language experimental condition]) that included three experimental conditions: phonological, semantic, and visual control. For each of these conditions, patients were required to 1) judge whether a visually presented pseudoword was composed of two syllables (e.g., "scrout" or "fruzon") during phonological condition; 2) perform a semantic categorization of words, i.e., determine whether a presented word was a living (such as "dog" or "dove") or nonliving (such as "table" or "car") entity during the semantic condition; and 3) judge whether a randomly arranged consonant string assembly was composed of capital or lowercase letters (e.g., "XTVNXD" or "xbxnbd") during the visual control (see Fig. 1 for protocol specification). Patients were instructed to answer as rapidly and accurately as possible by pressing a manual key with the left index finger for a "yes" and with the right index finger for a "no" response. Visual stimuli were delivered via Presentation software (Neurobehavioral Systems Inc.) and displayed on a 19-inch computer screen located
$60 \mathrm{~cm}$ away from the patient. Manual responses were recorded to assess behavioral performance. All patients underwent training before the experiment during a short session with different items from those presented during the experiment.

\section{Data Analysis \\ DCS Analysis}

All DCS effects during DCS naming or counting were carefully reviewed by a neurologist and/or neuropsychologist from the video-SEEG recordings of each patient. The video-SEEG review was performed in order to classify language interferences. Under the conventional speech/ language interference during DCS, we classify interferences as cognitive (including aphasia and paraphasic errors), motor (including dysarthria and speech motor arrest), and memory (including amnestic anomia).

To localize the sites from where language interference was elicited, we used a method similar to the one employed in a previous study by Sinai and collaborators: ${ }^{21}$ binary labeling of each explored cortical site and classification into sites that showed language symptoms (DCS+) and those that did not (DCS-). If an explored cortical site was common to two pairs of electrodes, the site was labeled DCSif any of these pairs did not induce language symptoms, or DCS+ if a language symptom was observed by DCS in pairs that included the electrode. As suggested by Sinai et al., ${ }^{21}$ this method is more relevant than a simple bipolar

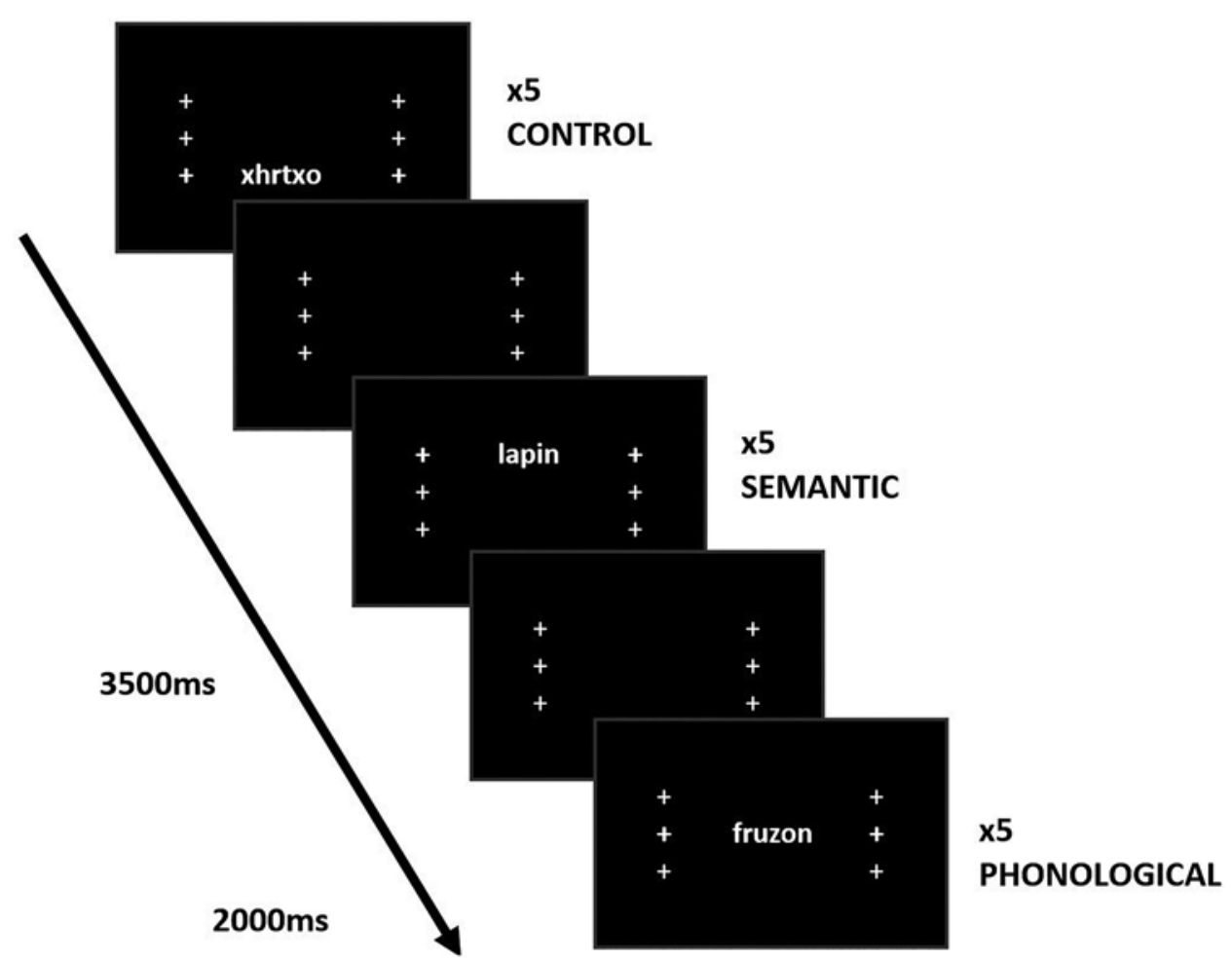

FIG. 1. Language paradigm for HFA mapping. Examples of experimental conditions: visual control, semantic, and phonological. Stimuli were presented on the bottom of the screen (visual control, consonant strings), on the top of the screen (semantic condition, words), or on the middle of the screen (phonological condition, pseudowords), allowing the patient to better remember the instructions for each condition. For each condition, 12 blocks were included, each composed of 5 trials. Sixty stimuli per condition were presented. Each stimulus lasted 2 seconds and stimuli were separated by a 3.5-second interval. 
labeling because DCS may impair the function supported by the cortex underlying each of the electrodes in a pair as well as by the cortex located between the electrode pair. ${ }^{22}$

\section{Induced HFA Analysis}

LEC-induced HFA $(50-150 \mathrm{~Hz})$ was defined by a preliminary time-frequency analysis of the SEEG data using a wavelet transform, ${ }^{23}$ performed using an in-house software package (ELAN, INSERM U1028 ${ }^{24}$ ) and based on previous studies in our group. ${ }^{18}$ Raw data were transformed into HFA time series with the following procedure. ${ }^{13,25}$ First, continuous SEEG signals were bandpass-filtered in multiple successive $10-\mathrm{Hz}$ wide-frequency bands (e.g., 10 bands from $50-60 \mathrm{~Hz}$ to $140-150 \mathrm{~Hz}$ ) using a zero-phaseshift, noncausal, finite impulse filter with $0.5-\mathrm{Hz}$ roll-off. Second, for each bandpass-filtered signal we computed the signal envelope using a standard Hilbert transform. ${ }^{26}$ The obtained envelope was downsampled to a sampling rate of $64 \mathrm{~Hz}$ (i.e., 1 sample every $15.625 \mathrm{msec}$ ). Third, for each band, this envelope signal (i.e., time-varying amplitude) was divided by its mean across the entire recording session and multiplied by 100 . This procedure yields instantaneous envelope values expressed in percentage of the mean. Fourth, the envelope signals (expressed in percentages) computed for each consecutive frequency band (the 10 bands of $10 \mathrm{~Hz}$ ) were averaged together to provide one single time series (HFA) across the entire session. By construction, the mean value of that time series across the recording session is equal to 100. And finally, the HFA time series was segmented, centered around each stimulus, and then averaged together for each stimulus category. Note that computing the Hilbert envelopes in $10-\mathrm{Hz}$ sub-bands and normalizing them individually before averaging over the broadband interval allows us to account for a bias toward the lower frequencies of the interval that would otherwise occur due to the $1 /$ frequency dropoff in amplitude.

All electrode data exhibiting pathological waveforms were discarded from the present study. This was achieved in collaboration with the medical staff and was based on visual inspection of the recordings and by systematically excluding data from any electrode site that was clinically found to be located within the seizure onset zone.

For each of the LEC task conditions, statistical analysis on HFAs was performed to test for significant increases or decreases compared to baseline activity (paired-sample Wilcoxon signed-rank test, followed by false discovery rate correction across all time samples). This allowed for quantitative definition of the onset time and duration of activation of specific recording sites. Thus, for each patient and for each cortical site, an HFA LEC map was computed and visually screened. Cortical sites showing epileptiform activity (based on visual inspection) were excluded from analysis. Hence, each cortical site showing significant statistical HFA was labeled as HFA+ and those without significant statistical HFA were labeled as HFA-. The visual inspection method used to evaluate HFA significance was performed by two independent observers of our team who were blinded to the rest of the patient data. Interrater agreement on detecting HFA activation was estimated from a total of 17,666 single-trial maps using Cohen's kappa coefficient, which showed a substantial agreement $(\kappa=0.75) .{ }^{27}$

\section{Comparison Between DCS and LEC-Induced HFA Maps}

Because DCS is considered the gold standard method for mapping eloquent language regions, we have only included anatomical sites in which DCS was applied. DCS results were compared to HFA results, according to the methodology proposed by Sinai et al..$^{21}$ More specifically, we determined: 1) the sensitivity, defined as the percentage of electrode sites that were positive for both methods (HFA+ and DCS+) among all DCS+ sites; 2) the specificity, calculated as the percentage of electrode sites that were negative for both methods (HFA- and DCS-) among all DCS- sites; 3) the positive predictive values (PPVs), calculated as the percentage of sites that were both HFA+ and DCS+ among all HFA+ sites, which allowed us to evaluate the probability that an HFA+ site showed language interference after DCS (DCS+); and 4) the negative predictive values (NPVs), computed as the percentage of sites that were both HFA- and DCS- among all HFA- sites, which allowed us to evaluate the probability that an HFA- site did not exhibit any language interference by DCS (DCS-).

\section{Results \\ DCS and LEC-Induced HFA Maps}

Overall, 3887 cortical sites were explored in the 42 patients (mean 92.5 sites, range $29-175$ sites per patient), of which 1914 (49\%) were electrically stimulated (mean 46 stimulated sites per patient, range $14-100$ per patient): $60 \%(\mathrm{n}=1144)$ of these stimulated sites were located in the left hemisphere and 40\% ( $\mathrm{n}=770)$ in the right hemisphere (Fig. 2, DCS).

Language interference responses (DCS+) were observed in $85(4.4 \%)$ of the 1914 stimulated cortical sites (Fig. 2, DCS+) in 27 of the 42 patients. As shown in Table 1 , functional MRI assessment for language was performed in only 20 of these 27 patients and showed that 17 of them presented with left hemisphere specialization, while 3 had bilateral representation for language.

Only 1 DCS+ site was observed during low-frequency stimulation $(1 \mathrm{~Hz}, 6 \mathrm{~mA})$, while the rest were observed during high-frequency stimulation $(50 \mathrm{~Hz}, 1-3 \mathrm{~mA})$. Furthermore, $83.9 \%(n=73)$ of the DCS+ sites were observed in the left hemisphere and $16.1 \%(n=14)$ in the right hemisphere. Regarding the left hemisphere, $67 \%(n=49)$ of the DCS+ sites were located in the temporal lobe, $27 \%(\mathrm{n}=$ $20)$ in the frontal lobe, and $5 \%(n=4)$ in the parietal lobe. For the right hemisphere, $67 \%(n=10)$ of the DCS+ sites were located in the frontal lobe and $33 \%(n=5)$ in the temporal lobe.

DCS+ symptoms (Fig. 3, DCS+) were labeled as 1) cognitive interference for $62.3 \%(\mathrm{n}=53$, of which 51 were observed in the left hemisphere and only 2 in the right hemisphere), 2) memory interferences for $24.7 \%(\mathrm{n}=11$, all in the left hemisphere), and 3) motor interferences for $19.5 \%$ ( $\mathrm{n}=21,9$ in the left and 12 in the right hemisphere).

All 42 patients exhibited induced HFA during LEC regardless of the experimental condition, at 771 of the 3887 recorded sites. Given that 1972 recorded sites were not stimulated, and thus provided information on HFA mapping only, we focused on the 1914 DCS sites. Thus, from 1914 DCS sites, 174 (9.1\%) exhibited LEC-induced HFA 


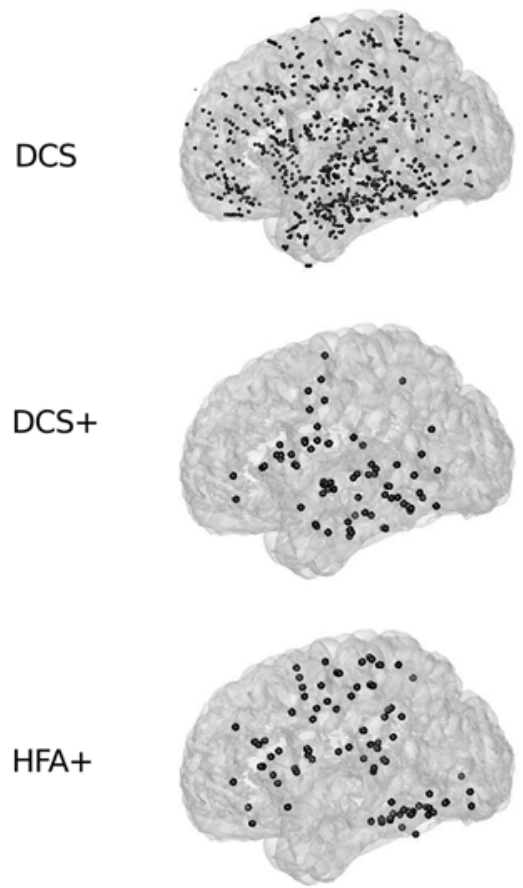

LH
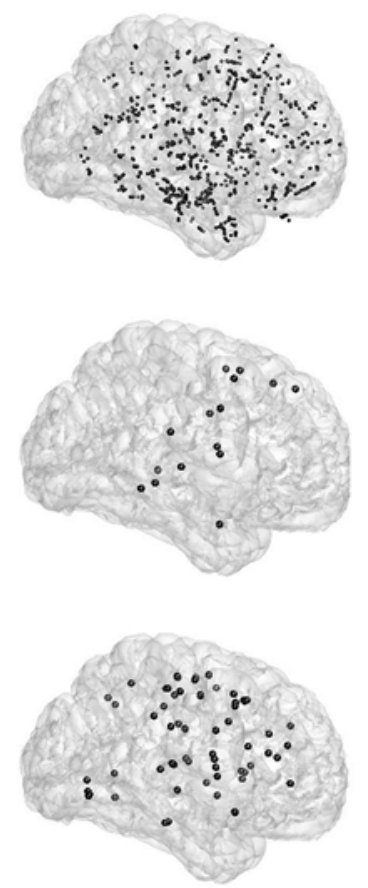

$\mathrm{RH}$
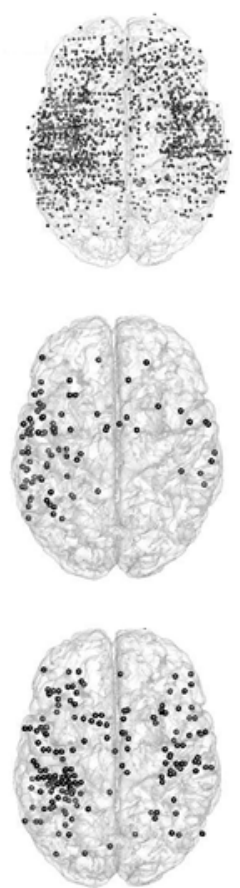

Top

FIG. 2. Spatial distribution of the stimulated sites. DCS: Spatial distribution of stimulated sites (1915 cortical sites) represented in the MNI space as lateral left side (left hemisphere, LH), lateral right side (right hemisphere, RH), and axial top view (Top). DCS+: Spatial distribution of DCS sites that led to a language response (DCS+ sites). HFAt: Spatial distribution of the cortical sites showing LEC-induced HFA in language conditions (HFA+ sites).

(HFA+) in 36 patients (mean $4.5 \pm 4.4$ per patient; Fig. 2, HFA+). HFA+ was observed for $63 \%(n=110)$ of the cases in the left hemisphere and for $37 \%(n=64)$ of the cases in the right hemisphere. In the left hemisphere, $42.7 \%$ (n $=47$ ) of the HFAs were located in the frontal lobe, $43.6 \%$ $(\mathrm{n}=48)$ in the temporal lobe, $10 \%(\mathrm{n}=11)$ in the parietal lobe, $2.7 \%(n=3)$ in the occipital lobe, and $0.9 \%(n=1)$ in the insular cortex. In the right hemisphere, $57.8 \%(\mathrm{n}=37)$ of the HFA+ sites were located in the frontal lobe, $10.9 \%$ $(\mathrm{n}=7)$ in the temporal lobe, 9.4\% $(\mathrm{n}=6)$ in the parietal lobe, $10.9 \%(\mathrm{n}=7)$ in the occipital lobe, and $10.9 \%(\mathrm{n}=7)$ in the insula.

Cortical sites labeled as HFA+ were represented by different selective induced responses according to LEC language conditions (Fig. 3, HFA+). Thus, 9.1\% of HFA+ sites presented with a selective induced response for the

DCS+
MOTOR
COGNITIVE
MEMORY

HFA+
PHON-SEM
PHON
SEM
VISU
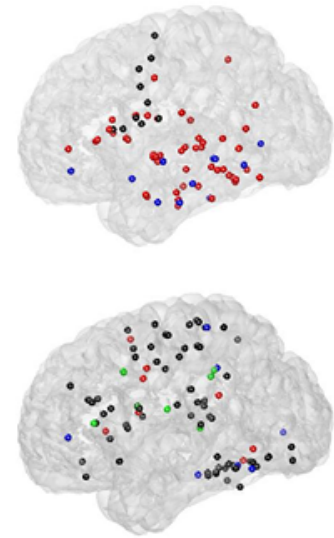

$\mathrm{LH}$
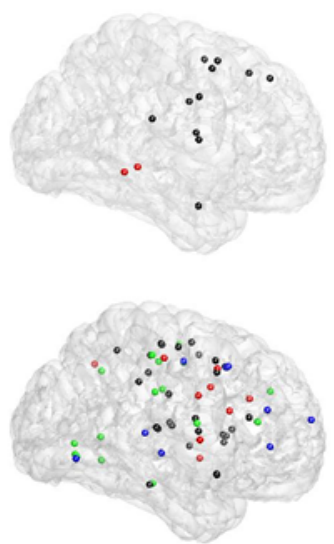

$\mathrm{RH}$
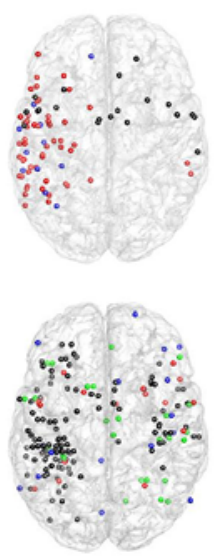

Top

FIG. 3. Spatial distribution of DCS and induced HFA sites for positive responses. DCS+ represents the spatial distribution according to each type of speech/language interference. HFA+ represents the spatial distribution according to each of the LEC task language conditions. PHON-SEM = phonological and semantic conditions; $\mathrm{PHON}=$ phonological condition; $\mathrm{SEM}=$ semantic condition; VISU = visual condition. Figure is available in color online only. 
semantic condition ( $\mathrm{n}=16$, with 7 sites in the left and 9 in the right hemisphere); $9.8 \%$ of HFA+ sites presented with a selective induced response for the phonological condition ( $\mathrm{n}=17$, with 8 in the left and 9 in the right hemisphere); $15.5 \%$ of HFA+ sites presented with a selective induced response for the visual condition $(n=27$, with 11 in the left and 16 in the right hemisphere); and the majority (65.5\%) of the HFA+ sites presented with coactivation for both phonological and semantic conditions $(n=114$, with 84 in the left and 30 in the right hemisphere).

\section{Comparison Between DCS and LEC-Induced HFA Maps}

The numbers of DCS sites showing positive and negative responses for each method (DCS and induced HFA, specifically for the semantic and phonological conditions) are presented in Table 2. A weak tetrachoric correlation ${ }^{28}$ (tcorr $=0.01)$ was observed between the results observed with DCS and those observed using LEC-induced HFA.

The sensitivity and specificity of induced HFA for language mapping relative to DCS were $8.9 \%$ and $92.4 \%$, respectively. Induced HFA showed a high NPV (95.9\%) but a low PPV (4.8\%), which suggests that induced HFA responses highly predict DCS responses only when negative responses are observed.

Only $7(0.36 \%)$ of the 1915 DCS sites were labeled as DCS+/HFAt: 2 were located in the left inferior frontal cortex (Brodmann's area [BA] 44 and 46), 3 in the left inferior temporal cortex (BA 20 and 37), 1 in the left supplementary motor area, and 1 in the right inferior parietal lobule (BA 40; see Table 3 for spatial distribution). Interestingly, 5 of these 7 DCS+/HFA+ sites showed HFA+ coactivation for both phonological and semantic LEC conditions. Three of these sites showed naming interference from a cognitive issue and the other 2 sites from a motor issue during DCS. Two of the DCS+/HFA+ sites showed a selective LEC condition response. The first, located at the level of the inferior temporal cortex (BA 20), presented with a selective HFA response for the semantic LEC condition. The second, located at the level of the inferior frontal cortex (BA 46), presented with a selective HFA response for the phonological LEC condition. These two cortical sites showed cognitive interference during DCS.

\section{Discussion}

We assessed the clinical relevance of the preoperative induced HFA for language mapping in patients with refractory epilepsy during SEEG recording. We showed that classic language areas can be mapped with both induced HFA and DCS methods. Induced HFA revealed a larger bilateral language network (frontotemporoparietal) compared to the left restricted DCS language network (frontotemporal). The major difference between the two language maps was observed in the parietal and temporal regions, in which induced HFA showed predominantly associative parietal regions, while DCS showed more associative temporal regions, including mesial temporal regions. This implies that DCS may be a better choice to map temporal regions. Differences between methods can be explained by their respective specificity. Indeed, DCS is a functional disrupting-inhibitory technique and therefore reveals
TABLE 2. Number of stimulation sites and positive and negative responses for DCS and induced HFA

\begin{tabular}{cccc}
\hline & DCS+ & DCS- & Total \\
\hline HFA+ & 7 & 139 & 146 \\
\hline HFA- & 72 & 1697 & 1769 \\
\hline Total & 79 & 1836 & 1915 \\
\hline
\end{tabular}

Number of positive (+) and negative (-) responses for each method: DCS and LEC-induced HFA (including semantic and phonological LEC conditions, i.e., without visual condition).

"essential" structures for a given cognitive process. Conversely, the induced HFA is a functional activating method that reveals the largest cortical network.$^{8,29}$ Still, subtle language dysfunctions triggered by DCS can be easily missed (not objective responses), leading to an underestimation of functional tissue. Consequently, language regions revealed by induced HFA are broader than, and partly overlap with, those defined by DCS. ${ }^{30}$ Furthermore, DCS may produce effects that are distant from the stimulated area, as the current may spread through axons or fibers to distant sites, ${ }^{31,32}$ as suggested by current density models showing that the focal current application influences distant areas. ${ }^{33}$

The major results of the present study suggested that language-related HFA predicted DCS language interference with a high specificity (92.4\%) and a high NPV $(95.9 \%)$. These results indicated that the absence of language-induced HFA makes a positive result by DCS very unlikely on these sites. Consequently, it would be possible to first record the induced HFA and map the language, and then apply DCS only as a second step. Precious time could be saved, and one can avoid stimulating sites that are irrelevant for language. These results are consistent with those provided by electrocorticography (ECOG) studies investigating similar frequency bands and that showed a specificity varying from $63 \%$ to $85 \% .^{21,34-36}$ For instance, Sinai et al., ${ }^{21}$ Arya et al. ${ }^{34}$ and Babajani-Feremi et al. ${ }^{35}$ showed high specificity $(84 \%, 81 \%$, and $85 \%$, respectively) using a naming task, as well as Towle et al. ${ }^{36}$ (57\%) using two language tasks (word repetition and word memory tasks).

Furthermore, and even if previous studies also showed lower sensitivity and PPVs than those observed for specificity and NPVs, our results suggest a weaker sensitivity and PPV than those reported in ECOG studies. We specifically showed a sensitivity of $8.9 \%$ and a PPV of $4.8 \%$, while previous studies ranged from $41 \%$ to $57 \%$ for sensitivity. ${ }^{9,26,32}$ Such differences could be explained by different factors, such as the method used during DCS (ECOG vs SEEG), HFA computation (including the frequency range used for HFA computation), and the language tasks used during DCS and HFA.

First, SEEG DCS is focal, because stimulation is applied in a bipolar manner between contacts that are only distant by $2.5 \mathrm{~mm}$ (center to center). Still, the current intensity used for functional mapping with grids is usually higher (up to $15 \mathrm{~mA}$ ) than with SEEG electrodes (up to 3-4 mA), consistent with the safety recommendation. ${ }^{37}$ Previous studies suggested that DCS effects depend on several factors; ${ }^{11,36,38}$ thus, it remains uncertain whether the DCS intensity applied in our study was sufficient to show 
TABLE 3. DCS sites showing both DCS+ and HFA+ responses

\begin{tabular}{|c|c|c|c|c|c|c|c|c|c|c|}
\hline \multirow[b]{2}{*}{ Pt No. } & \multirow[b]{2}{*}{$\mathrm{LL}$} & \multirow{2}{*}{$\begin{array}{l}\text { Electrode } \\
\text { Hemisphere }\end{array}$} & \multirow[b]{2}{*}{ Anatomical Label } & \multirow[b]{2}{*}{ BA } & \multicolumn{3}{|c|}{ MNI Coordinates } & \multirow[b]{2}{*}{$\mathrm{HFA}+$} & \multirow[b]{2}{*}{ DCS+ } & \multirow{2}{*}{$\begin{array}{l}\text { Frequency } \\
\text { (intensity) }\end{array}$} \\
\hline & & & & & $x$ & $\mathrm{y}$ & z & & & \\
\hline 2 & Bilat & $\mathrm{Rt}$ & Inferior parietal cortex & 40 & 63.9 & -25.8 & 23.3 & $\mathrm{P}+, \mathrm{S}+$ & Motor & $50 \mathrm{~Hz}(3 \mathrm{~mA})$ \\
\hline 4 & Lt & $\mathrm{Lt}$ & Rostral inferior temporal cortex & 20 & -46.1 & -29.4 & -19 & $\mathrm{P}-, \mathrm{S}+$ & Cog & $50 \mathrm{~Hz}(2 \mathrm{~mA})$ \\
\hline 4 & Lt & $\mathrm{Lt}$ & Inferior temporal cortex & 37 & -56.8 & -50.5 & -15.2 & $\mathrm{P}+\mathrm{S}+$ & $\operatorname{Cog}$ & $50 \mathrm{~Hz}(2 \mathrm{~mA})$ \\
\hline 12 & NA & $\mathrm{Lt}$ & Inferior frontal cortex & 44 & -50 & 10 & 7.1 & $\mathrm{P}+\mathrm{S}+$ & Cog & $50 \mathrm{~Hz}(2 \mathrm{~mA})$ \\
\hline 26 & $\mathrm{Lt}$ & $\mathrm{Lt}$ & Supplementary motor area & 6 & -6 & -3 & 52.8 & $\mathrm{P}+\mathrm{S}+$ & Motor & $50 \mathrm{~Hz}(1.6 \mathrm{~mA})$ \\
\hline 31 & $\mathrm{Lt}$ & $\mathrm{Lt}$ & Inferior frontal cortex & 46 & -49.2 & 43.4 & 1.9 & $\mathrm{P}+, \mathrm{S}-$ & Cog & $50 \mathrm{~Hz}(3 \mathrm{~mA})$ \\
\hline 40 & NA & $\mathrm{Lt}$ & Inferior temporal cortex & 37 & -43.5 & -46.6 & -12.9 & $\mathrm{P}+\mathrm{S}+$ & Cog & $50 \mathrm{~Hz}(2 \mathrm{~mA})$ \\
\hline
\end{tabular}

DCS+ = type of interference shown during DCS: cognitive (Cog) or motor (Motor) interference; HFA+ = phonological (P), semantic (S) conditions of LEC.

significant language disturbances. To our knowledge, there have been no studies that explored the "optimal" DCS parameters to apply for language or, more generally, for cognitive mapping (see Britton ${ }^{38}$ ). Importantly, some authors used higher thresholds than in our study (up to $8 \mathrm{~mA}$ ) for language mapping but a shorter pulse of $0.3 \mathrm{msec}$, leading to the same charge density delivered in this study. In addition, HFA+ was recorded in a larger number of cortical sites, but only approximately half of them were stimulated and included in the analysis, as we chose DCS as a gold standard; therefore, this also could have influenced the sensitivity/specificity of our results.

Second, the difference between previous studies is the frequency bands used within the broad range of HFA: for example, $80-100 \mathrm{~Hz}$ in the study by Sinai et al. ${ }^{21} 70-100$ $\mathrm{Hz}$ by Towle et al., ${ }^{36} 70-116 \mathrm{~Hz}$ by Arya et al., ${ }^{34} 76-200$ $\mathrm{Hz}$ by Miller et al., ${ }^{39}$ and $50-150 \mathrm{~Hz}$ in our study. It is important to note that currently no study, to our knowledge, has been performed to determine which frequency bands are the best to predict DCS results. Bauer et al. ${ }^{40}$ performed language mapping from several language tasks using different frequency bands. They showed that lower frequencies appeared more sensitive for DCS than higher frequencies, which could be related to the high task and stimulation sensitivity observed for the induced HFA (see Lachaux et al. ${ }^{8}$ ). Indeed, lower frequencies could be less area specific and thus induce a better relationship to DCS results. This could be linked with recent studies suggesting that DCS effects could be outside the stimulated current field. Therefore, functional deficits (induced by DCS) of a cortical region may reflect dysfunction of a large-scale network $^{41,42}$ and DCS language deficits may result from the perturbation of this language network instead of solely the stimulated site (see David et al. ${ }^{43}$ and Matsumoto et al. ${ }^{44}$ ). Thus, DCS could have effects outside the immediate area of stimulation and explain the low relationship with induced HFA results, which are believed to be spatially focal (e.g., Lachaux et al. ${ }^{8}$ ). Finally, even if the majority of studies used different tasks during DCS and HFA measures, Sinai et al. ${ }^{21}$ used the naming task during the two methods and the sensitivity (42\%) was also lower compared with the specificity (84\%). In the present study, the language tasks used during DCS and HFA were significantly different. This can be an important limitation of the present study because the cerebral regions involved in each task may differ, as different linguistic processes are used to trigger responses. ${ }^{45}$ Thus, our very low sensitivity (8.9\%) and PPV (4.8\%) could be explained by the high stimuli and task sensitivity of the induced HFA map, which is reflected by a very focal spatial activity, in contrast to recent results that suggested a more diffuse cortical effect of DCS.

A more direct way to assess the clinical relevance of induced HFA would be to evaluate the impact of HFA+ site resection. According to this evaluation, if the HFA is a relevant method for functional brain mapping, we could hypothesize that resection would be associated with language decline if the resection included an HFA+ site. In the present study, we presented a limited number of functional sites resected (approximately 1.2\% of sites in each patient), therefore not allowing us to evaluate this hypothesis directly.

\section{Conclusions}

DCS language mapping appears to be more appropriate for an extensive temporal mapping than induced HFA mapping. Furthermore, induced HFA should be used as a complement to DCS to preselect the number of stimulated sites during DCS by omitting those reported as HFA-. This may be a considerable advantage because it allows a reduction in the duration of the stimulation procedure. We discuss several parameters to be used for each method, and we interpret our results in relation to previous results reported in ECOG studies.

\section{Highlights}

- HFA predicts language interference observed during DCS with high specificity (92.4\%) and a high negative predictive value $(95.9 \%)$.

- Task-induced HFA represented a useful complement to DCS by strongly predicting the ineffectiveness of stimulation procedures.

- DCS may be a better choice to map temporal language regions.

\section{Acknowledgments}

We thank all patients, the staff of the Grenoble Neurological Hospital epilepsy unit, and Patricia Boschetti, Virginie Cantale, Marie Pierre Noto, and Chrystelle Mosca for their support. The research leading to these results has received funding from the 
National ANR FORCE (grant no. ANR-13-TECS-0013-01), ANR REORG (grant no. ANR-17-CE28-0015-01), NeuroCoG IDEX UGA ("Investissements d'avenir program," grant no. ANR-15IDEX-02), and Institut Universitaire de France.

\section{References}

1. Kahane P, Dubeau F. Intracerebral depth electrodes electroencephalography (stereoencephalography). In: Ebersole JS, ed. Current Practice of Clinical Electroencephalography. 4th ed. Wolters Kluwer; 2014:393-441

2. Kahane P, Tassi L, Francione S, et al. Electroclinical manifestations elicited by intracerebral electric stimulation "shocks" in temporal lobe epilepsy [in French]. Neurophysiol Clin. 1993;23(4):305-326.

3. Penfield W, Jasper H: Epilepsy and the Functional Anatomy of the Human Brain. Little Brown \& Co; 1954.

4. Borchers S, Himmelbach M, Logothetis N, Karnath H-O. Direct electrical stimulation of human cortex-the gold standard for mapping brain functions? Nat Rev Neurosci. 2011;13(1):63-70.

5. Desmurget M, Song Z, Mottolese C, Sirigu A. Re-establishing the merits of electrical brain stimulation. Trends Cogn Sci. 2013;17(9):442-449.

6. Hamberger MJ. Object naming in epilepsy and epilepsy surgery. Epilepsy Behav. 2015;46:27-33.

7. Vidal JR, Hamame CM, Jerbi K, et al. Localizing cognitive functions in epilepsy with intracranial gamma-band dynamic responses. In: Helmstaedter C, Hermann B, Lassonde M, et al., eds. Neuropsychology in the Care of People With Epilepsy. John Libbey Eurotext; 2011.

8. Lachaux J-P, Axmacher N, Mormann F, et al. High-frequency neural activity and human cognition: past, present and possible future of intracranial EEG research. Prog Neurobiol. 2012;98(3):279-301.

9. Helmstaedter C. Neuropsychological aspects of epilepsy surgery. Epilepsy Behav. 2004;5(suppl 1):S45-S55.

10. Sherman EMS, Wiebe S, Fay-McClymont TB, et al. Neuropsychological outcomes after epilepsy surgery: systematic review and pooled estimates. Epilepsia. 2011;52(5):857-869.

11. Juphard A, Vidal JR, Perrone-Bertolotti M, et al. Direct evidence for two different neural mechanisms for reading familiar and unfamiliar words: an intra-cerebral EEG study. Front Hum Neurosci. 2011;5:101.

12. Mainy N, Kahane P, Minotti L, et al. Neural correlates of consolidation in working memory. Hum Brain Mapp. 2007;28(3):183-193.

13. Perrone-Bertolotti M, Kujala J, Vidal JR, et al. How silent is silent reading? Intracerebral evidence for top-down activation of temporal voice areas during reading. J Neurosci. 2012;32(49):17554-17562.

14. Arya R, Horn PS, Crone NE. ECoG high-gamma modulation versus electrical stimulation for presurgical language mapping. Epilepsy Behav. 2018;79:26-33.

15. Arya R, Ervin B, Dudley J, et al. Electrical stimulation mapping of language with stereo-EEG. Epilepsy Behav. 2019;99:106395.

16. Kahane P, Minotti L, Hoffmann D, et al. Invasive EEG in the definition of the seizure onset zone: depth electrodes. In: Rosenow F, Lüders HO, eds. Handbook of Clinical Neurophysiology. Elsevier; 2003:109-133

17. Deman P, Bhattacharjee M, Tadel F, et al. IntrAnat electrodes: a free database and visualization software for intracranial electroencephalographic data processed for case and group studies. Front Neuroinform. 2018;12:40.

18. Jerbi K, Ossandón T, Hamamé CM, et al. Task-related gamma-band dynamics from an intracerebral perspective: review and implications for surface EEG and MEG. Hum Brain Mapp. 2009;30(6):1758-1771.
19. Lachaux JP, Rudrauf D, Kahane P. Intracranial EEG and human brain mapping. J Physiol Paris. 2003;97(4-6):613-628.

20. Deloche G, Hannequin D. Test de dénomination orale d'images DO80. ECPA; 1997.

21. Sinai A, Bowers CW, Crainiceanu CM, et al. Electrocorticographic high gamma activity versus electrical cortical stimulation mapping of naming. Brain. 2005;128(Pt 7):1556-1570.

22. Nathan SS, Sinha SR, Gordon B, et al. Determination of current density distributions generated by electrical stimulation of the human cerebral cortex. Electroencephalogr Clin Neurophysiol. 1993;86(3):183-192.

23. Tallon-Baudry C, Bertrand O, Delpuech C, Permier J. Oscillatory $\gamma$-band $(30-70 \mathrm{~Hz})$ activity induced by a visual search task in humans. J Neurosci. 1997;17(2):722-734.

24. Aguera P-E, Jerbi K, Caclin A, Bertrand O. ELAN: a software package for analysis and visualization of MEG, EEG, and LFP signals. Comput Intell Neurosci. 2011;2011:158970.

25. Ossandón T, Jerbi K, Vidal JR, et al. Transient suppression of broadband gamma power in the default-mode network is correlated with task complexity and subject performance. $J$ Neurosci. 2011;31(41):14521-14530.

26. Le Van Quyen M, Staba R, Bragin A, et al. Large-scale microelectrode recordings of high-frequency gamma oscillations in human cortex during sleep. J Neurosci. 2010;30(23):7770-7782.

27. Cuisenier P, Robin A, Job A-S, et al. Reproductibilité de l'analyse visuelle de cartes d'activité à hautes fréquences (HFA) évaluant les réseaux fonctionnels cognitifs en SEEG. Neurophysiol Clin Neurophysiol. 2016;46:222.

28. Divgi DR. Calculation of the tetrachoric correlation coefficient. Psychometrika. 1979;44:169-172.

29. Crone NE, Sinai A, Korzeniewska A. High-frequency gamma oscillations and human brain mapping with electrocorticography. Prog Brain Res. 2006;159:275-295.

30. Brown EC, Rothermel R, Nishida M, et al. In vivo animation of auditory-language-induced gamma-oscillations in children with intractable focal epilepsy. Neuroimage. 2008;41(3):1120-1131.

31. Blume WT, Jones DC, Pathak P. Properties of after-discharges from cortical electrical stimulation in focal epilepsies. Clin Neurophysiol. 2004;115(4):982-989.

32. Lesser RP, Lüders H, Klem G, et al. Cortical afterdischarge and functional response thresholds: results of extraoperative testing. Epilepsia. 1984;25(5):615-621.

33. Ranck JB Jr. Which elements are excited in electrical stimulation of mammalian central nervous system: a review. Brain Res. 1975;98(3):417-440.

34. Arya R, Wilson JA, Fujiwara H, et al. Presurgical language localization with visual naming associated ECoG high- gamma modulation in pediatric drug-resistant epilepsy. Epilepsia. 2017;58(4):663-673.

35. Babajani-Feremi A, Wheless JW, Papanicolaou JW, et al. Spatial-temporal functional mapping of language at the bedside with electrocorticography. Neurology. 2016;87(24):2604.

36. Towle VL, Yoon H-A, Castelle M, et al. ECoG gamma activity during a language task: differentiating expressive and receptive speech areas. Brain. 2008;131(Pt 8):2013-2027.

37. Shannon RV. A model of safe levels for electrical stimulation. IEEE Trans Biomed Eng. 1992;39(4):424-426.

38. Britton JW. Electrical stimulation mapping with stereo-EEG electrodes. J Clin Neurophysiol. 2018;35(2):110-114.

39. Miller KJ, Abel TJ, Hebb AO, Ojemann JG. Rapid online language mapping with electrocorticography. J Neurosurg Pediatr. 2011;7(5):482-490.

40. Bauer PR, Vansteensel MJ, Bleichner MG, et al. Mismatch between electrocortical stimulation and electrocorticography frequency mapping of language. Brain Stimul. 2013;6(4):524-531.

41. Mandonnet E, de Witt Hamer P, Duffau H. MRI screening 
for glioma: a preliminary survey of healthy potential candidates. Acta Neurochir (Wien). 2016;158(5):905-906.

42. Mandonnet E, Winkler PA, Duffau H. Direct electrical stimulation as an input gate into brain functional networks: principles, advantages and limitations. Acta Neurochir(Wien). 2010;152(2):185-193.

43. David O, Blauwblomme T, Job A-S, et al. Imaging the seizure onset zone with stereo-electroencephalography. Brain. 2011;134(Pt 10):2898-2911.

44. Matsumoto R, Nair DR, LaPresto E, et al. Functional connectivity in the human language system: a cortico-cortical evoked potential study. Brain. 2004;127(Pt 10):2316-2330.

45. Baciu M, Perrone-Bertolotti M. What do patients with epilepsy tell us about language dynamics? A review of fMRI studies. Rev Neurosci. 2015;26(3):323-341.

\section{Disclosures}

The authors report no conflict of interest concerning the materials or methods used in this study or the findings specified in this paper.

\section{Author Contributions}

Conception and design: Perrone-Bertolotti, Cuisenier, Minotti, Lachaux, Kahane. Acquisition of data: Perrone-Bertolotti, Cuisenier, Testud, Minotti, Deman, Hoffmann, Kahane. Analysis and interpretation of data: Perrone-Bertolotti, Cuisenier, Testud, El Bouzaïdi Tiali, Martineau, Job, Deman, Bhattacharjee, Hoffmann, Kahane. Drafting the article: Perrone-Bertolotti, Cuisenier, Testud, Minotti, El Bouzaïdi Tiali, Martineau, Job, Trebuchon, Deman, Lachaux, Baciu, Kahane. Critically revising the article: Perrone-Bertolotti, Cuisenier, Minotti, Kahane. Reviewed submitted version of manuscript: Perrone-Bertolotti, Cuisenier, Minotti, Kahane. Approved the final version of the manuscript on behalf of all authors: Perrone-Bertolotti. Statistical analysis: Perrone-Bertolotti, Cuisenier. Administrative/techni$\mathrm{cal} /$ material support: Perrone-Bertolotti, Minotti, Kahane. Study supervision: Perrone-Bertolotti, Kahane.

\section{Correspondence}

Marcela Perrone-Bertolotti: UFR Sciences de l'Homme et de la Société, Bâtiment Michel Dubois, Grenoble, France. marcela. perrone-bertolotti@univ-grenoble-alpes.fr. 